\title{
Serum Selenoprotein $P$ level is not indicative of Insulin Resistance in Children and Adolescents with Type 1 Diabetes Mellitus: Single Center Experience
}

\author{
Hend Mehawed Soliman', Balsam Sherif Fahmy ${ }^{2 *}$, Selim Mohamed Abdelkader ${ }^{3}$, \\ Samah A. Hassanein ${ }^{1}$ \\ 1 Pediatrics Department, Faculty of Medicine, Cairo University, Egypt; ziad.alaa@yahoo.com, \\ s_ahmed202020@yahoo.com \\ 2 Clinical and Chemical Pathology Department, Faculty of Medicine, Cairo University, Egypt. \\ 3 Pediatrics Department, El Menshawy General Hospital, Tanta, Egypt; mohamed.seleem90@yahoo.com \\ * Correspondence: balsamsherif338@yahoo.com \\ Received: 28/11/2021; Accepted: 20/12/2021; Published online: 27/12/2021.
}

\begin{abstract}
:
Background: Selenium has an anti-diabetic action as it an insulin-mimetic and antioxidant nutrient. Selenoprotein $\mathrm{P}(\mathrm{SeP})$ is an extracellular glycoprotein, that was linked to insulin resistance (IR).

Aim: To validate of serum $\mathrm{SeP}$ as a measure for insulin resistance in patients with type 1 diabetes mellitus (T1DM).

Methods: This prospective case-control study included 45 children and adolescents with T1DM and 45 healthy children and adolescents. Serum SeP was measured by ELISA and compared to estimated glucose disposal rate (eGDR) as a measure of insulin resistance.

Results: Mean \pm SD of SeP level was higher in T1DM patients than control group $(59.78 \pm 59.38$ $\mathrm{ng} / \mathrm{ml}, 55.57 \pm 7.6 \mathrm{ng} / \mathrm{ml}$, respectively), this difference was statistically insignificant $(\mathrm{p}=0.642)$. SeP demonstrated significant positive correlations with duration of diabetes $(r=0.413, p=0.005)$, high density lipoproteins $(r=0.496, p=0.001)$ and glycosylated hemoglobin $(r=0.357, p=0.016)$. There were statistically significant differences in eGDR between cases and controls $(7.78 \pm 3.08$ $\mathrm{mg} / \mathrm{kg} / \mathrm{min}, 12.53 \pm 0.91 \mathrm{mg} / \mathrm{kg} / \mathrm{min}$, respectively, $(\mathrm{p}=0.001)$. There was no correlation between SeP level and eGDR values (IR indicator).

Conclusion: Serum SeP level in T1DM patients was not indicative of IR. Higher serum SeP level are associated with longer duration and poor control of T1DM.
\end{abstract}

Level of Evidence of Study: IIB (1).

Keywords: Diabetes Mellitus, Insulin resistance, Selenoprotein P, estimated glucose disposal rate.

Abbreviations: eGDR: Estimated glucose disposal rate; HbA1c: glycosylated hemoglobin; IR: insulin resistance; SeP: Selenoprotein P; T1DM: Type 1 diabetes mellitus;.

\section{Introduction}

Absolute insulin deficiency is pathogenic basis of type 1 diabetes mellitus (T1DM); however development of insulin resistance (IR) may occur in T1DM patients (1). Children with T1DM with IR are more prone to chronic complications (2). Insulin sensitivity is measured effectively using the euglycemic-hyperinsulinemic clamp; but it is not readily available in the clinical setting (3). IR can also be assessed using estimated glucose disposal rate (eGDR) which is an effective practical measure, with higher IR in individuals who had lower eGDR levels. Glycosolated hemoglobin (HbA1c), waist circumference and presence or absences of hypertension are the indicators used in eGDR calculation (4). Selenium has an anti-diabetic action as it an insulin-mimetic and antioxidant nutrient, which might be reversed by high selenium intake (5). Selenoprotein $\mathrm{P}(\mathrm{SeP})$ is an extracellular glycoprotein, regulated by dietary selenium supplementation (6). Most tissues express SeP; however, its majority is expressed in hepatocytes (7). Insulin impairs release of SeP from the liver in healthy individuals with normal insulin sensitivity. While in those with IR; a change in SeP level occurs due to disrupting of this feedback 
mechanism. This was thought to cause hyperglycemia in those patients and IR (8). SeP impairs insulin receptors function and decreases insulin- evoked cellular uptake of glucose. SeP was reported to induce IR in the hepatocytes through disrupting mitogen-activated protein kinase (MAPK) pathway. Also excess SeP limits insulin production through decreasing the pancreatic B cells number (9). The goal of our study was to assess levels of serum SeP in children with T1DM and to detect the relationship of SeP with glucose control, serum lipids and IR compared to eGDR.

\section{Subjects and Methods}

This was a prospective case-control study carried out on 45 children and adolescents with T1D and 45 healthy age and sex-matched children serving as controls. Patients were recruited from the Diabetes, Endocrine \& Metabolism Pediatric Unit (DEMPU) outpatient clinic, Cairo University while controls were recruited from the general outpatient clinics, The Children's Hospital, Cairo University. The Pediatric Department and Scientific Ethics Committee of Faculty of Medicine, Cairo University (code: MS-229-2020) had approved this study that abided by the Declaration of Helsinki for the protection of human subjects (10).

\section{Participants}

Children with T1DM of duration more than 1 year and age between 8-18 years were included in this study. Patients who suffered other endocrinal diseases (e.g. celiac disease, thyroid disease, adrenal disorder), conditions that may affect serum SeP levels (e.g. cancer, heart disease, infectious viral or inflammatory disease) or those taking selenium supplements or drugs that may affect serum SeP level (e.g. aminoglycosides, cisplatin, clozapine, corticosteroids \& valproic acid), were not included in the study.

\section{Methods}

All participants in the study were subjected to a full history-taking including age, gender, age at time of diagnosis, presenting symptoms and history of drug intake that can affect serum SeP levels. Clinical examination included anthropometric measures (height, height SDS, weight, weight SDS, body mass index (BMI), BMI SDS, hip circumference, waist circumference, and waist-hip ratio). Waist circumference was assessed at the half of the distance between the lowest rib and the iliac crest (11). Blood pressure (BP) was measured and expressed as centiles for age and gender (12). Results of fasting blood glucose, HbA1c and fasting triglycerides, total cholesterol, low density lipoprotein (LDL) and high density lipoprotein (HDL) were recorded. Insulin resistance was calculated using eGDR according to the following equation: eGDR $(\mathrm{mg} / \mathrm{kg} / \mathrm{min})=(-0.551 \times \mathrm{HbA} 1 \mathrm{c})+(-3.407 \times \mathrm{HTN})+(-0.09 \times \mathrm{WC})+21.158$, where $\mathrm{WC}=$ waist circumference measured in $\mathrm{cm}$ and HTN is the presence of hypertension ( $0=$ no, $1=y e s)(13)$.

\section{Selenoprotein P measurement}

Venous blood samples were collected from all participants and tested for fasting blood glucose (FBG), HbA1c and serum lipids and serum SeP. Samples for SeP were centrifuged at a speed of 2000-3000 $\mathrm{rpm}$ for $15 \mathrm{~min}$ after leaving at room temperature for 10-20 min. The supernatant was removed and stored at $-20^{\circ} \mathrm{c}$ till time of assay. Serum SeP was assayed using commercially available ELISA kit (Cat No: SG-10784, sinoGeneClon Biotech Co., Ltd, HangZhou, China) according to the manufacturer instructions. The standard preparation supplied with the assay was used to plot standard curve. The optical density readings at $450 \mathrm{~nm}$ were converted to concentrations in $\mathrm{ng} / \mathrm{ml}$ after the reaction.

\section{Statistical Analysis}

Statistical Package of Social Science (SPSS) Software program (version 22.0) was used to analyze data. Frequencies and percentages were used to express qualitative data while mean and standard deviation were used to express quantitative data if parametric. Median and range were used to express quantitive data if non-parametric. Student t-test and one-way ANOVA test (if parametric) and Chi square ( $\left.x^{2}\right)$ were used in comparison between groups. Linear Correlation Coefficient was calculated to assess relation between quantitative variables. Significant $p$ value was $<0.05$. 


\section{Results}

The study included 45 T1DM patients and 45 sex and age-matched healthy controls. The mean $( \pm \mathrm{SD})$ age of the cases was $11.2 \pm 2.4$ years while the mean $( \pm \mathrm{SD})$ age of the controls was $11.48 \pm 2.6$ years. Twenty four cases (53.3 \%) were females and 21 (46.7\%) were males. Demographic, clinical, and laboratory data of the studied groups are summarized in Table1. Height SDS was significantly lower $(p=0.001)$, while BMI SDS, systolic and diastolic blood pressure percentiles were higher in cases than in controls with significant $\mathrm{p}$ value $(p=0.003$, 0.011, 0.007 and 0.001 respectively) as shown in Table 1 . HbA1c, total cholesterol, triglycerides, LDL, FBS and $2 \mathrm{~h}$ PPs were significantly higher $(p=0.001)$ while HDL was significantly lower $(P=0.001)$ in cases than in controls. These findings reflect the uncontrolled blood glucose levels and dyslipidemia that are common with T1DM, risk factors that predispose to cardiovascular diseases as in table 1. Cases had significantly lower eGDR than controls ( $7.78 \pm 3.08$ vs $12.53 \pm 0.91$ $\mathrm{mg} / \mathrm{kg} / \mathrm{min})(\mathrm{p}=0.001)$ reflecting insulin resistance in cases as shown in Table 2.

Table 1: Demographic, clinical and laboratory data of studied groups.

\begin{tabular}{|c|c|c|c|c|}
\hline \multicolumn{2}{|c|}{ Demographic data } & $\begin{array}{c}\text { Cases } \\
(\text { No. }=45)\end{array}$ & $\begin{array}{c}\text { Control } \\
(\text { No. = 45) }\end{array}$ & $\begin{array}{c}\mathbf{P} \\
\text { value }\end{array}$ \\
\hline \multicolumn{2}{|c|}{ Age (year) } & $11.20 \pm 2.40$ & $11.48 \pm 2.60$ & 0.603 \\
\hline \multirow{2}{*}{$\begin{array}{l}\text { Gender } \\
\text { No. (\%) }\end{array}$} & Male & $21(46.7 \%)$ & $22(48.9 \%)$ & \multirow{2}{*}{0.833} \\
\hline & Female & $24(53.3 \%)$ & $23(51.1 \%)$ & \\
\hline \multirow{2}{*}{$\begin{array}{l}\text { Family } \\
\text { History } \\
\text { No. (\%) }\end{array}$} & Positive & $27(60 \%)$ & $21(46.7 \%)$ & \multirow{2}{*}{0.205} \\
\hline & Negative & $18(40 \%)$ & $24(53.3 \%)$ & \\
\hline \multirow{2}{*}{$\begin{array}{l}\text { Puberty } \\
\text { No. (\%) }\end{array}$} & Yes & $6(13.3 \%)$ & $10(22.2 \%)$ & \multirow{2}{*}{0.270} \\
\hline & No & $39(86.7 \%)$ & $35(77.8 \%)$ & \\
\hline \multicolumn{2}{|l|}{ Weight SDS } & $1.20 \pm 1.79$ & $0.98 \pm 0.52$ & 0.432 \\
\hline \multicolumn{2}{|l|}{ Height SDS } & $-1.55 \pm 1.06$ & $-0.55 \pm 0.65$ & 0.001 \\
\hline \multicolumn{2}{|c|}{ BMI SDS } & $0.60 \pm 2.28$ & $-1.01 \pm 0.62$ & 0.003 \\
\hline \multicolumn{2}{|c|}{ Waist / Hip ratio } & $0.87 \pm 0.10$ & $0.85 \pm 0.02$ & 0.180 \\
\hline \multicolumn{2}{|c|}{ Systolic percentile (\%) } & $73.42 \pm 28.20$ & $59.80 \pm 17.78$ & 0.007 \\
\hline \multicolumn{2}{|c|}{ HbA1c (\%) } & $10.46 \pm 1.97$ & $4.78 \pm 0.31$ & 0.001 \\
\hline \multicolumn{2}{|l|}{ FBS (mg/dl) } & $146.31 \pm 33.52$ & $102.60 \pm 9.74$ & 0.001 \\
\hline \multicolumn{2}{|c|}{ 2h PPs (mg/dl) } & $275.56 \pm 93.46$ & $163.93 \pm 10.89$ & 0.001 \\
\hline \multicolumn{2}{|c|}{ Cholesterol (mg/dl) } & $193.67 \pm 45.43$ & $153.04 \pm 7.04$ & 0.001 \\
\hline \multicolumn{2}{|c|}{ Triglycerides $(\mathrm{mg} / \mathrm{dl})$} & $105.47 \pm 25.18$ & $82.64 \pm 4.71$ & 0.001 \\
\hline \multicolumn{2}{|c|}{ HDL $(\mathrm{mg} / \mathrm{dl})$} & $38.18 \pm 15.47$ & $53.78 \pm 3.32$ & 0.001 \\
\hline \multicolumn{2}{|l|}{$\mathrm{LDL}(\mathrm{mg} / \mathrm{dl})$} & $112.89 \pm 21.31$ & $95.67 \pm 4.81$ & 0.001 \\
\hline
\end{tabular}

BMI (body Mass Index), Systolic blood pressure, Diastolic blood pressure, EGDR (Estimated Glucose Disposal Rate), HDL (High density lipoprotein), LDL (Low density lipoprotein), FBS (Fasting blood sugar), $2 \mathrm{~h}$ PPs (2h post prandial sugar); $\mathrm{P}$ value $<0.05$ : Statistically significant.

Table 2: Comparison between Cases and Control groups regarding eGDR and SeP levels.

\begin{tabular}{|c|c|c|c|c|}
\hline & & $\begin{array}{l}\text { Cases group } \\
(\text { No. }=45)\end{array}$ & $\begin{array}{c}\text { Control group } \\
(\text { No. }=45)\end{array}$ & $\begin{array}{c}\mathbf{P} \\
\text { value }\end{array}$ \\
\hline eGDR (mg/kg/min) & & $\begin{array}{l}7.78 \pm 3.08 \\
3.14-12.28\end{array}$ & $\begin{array}{c}12.53 \pm 0.91 \\
10.4-13.59\end{array}$ & 0.001 \\
\hline Selenoprotein P (ng/ml) & $\begin{array}{c}\text { Mean } \pm \mathrm{SD} \\
\text { Range }\end{array}$ & $\begin{array}{c}59.78 \pm 60.05 \\
(12-312) \\
\end{array}$ & $\begin{array}{c}55.57 \pm 7.60 \\
45-75.5 \\
\end{array}$ & 0.642 \\
\hline
\end{tabular}

eGDR in T1DM patients was negatively correlated with significant $\mathrm{p}$ value with their weight SDS $(r=-0.727, p=0.001)$, BMI SDS $(r=-0.883, p=0.001)$, waist/hip ratio $(r=-0.746, p=0.001)$, revealing that higher body weight and BMI was associated with insulin resistance shown in Table 3. In addition, eGDR showed statistically significant negative correlations with systolic blood pressure percentile $(r=-0.683, p=0.001)$ and diastolic blood pressure percentile $(r=-0.725$, 
$\mathrm{p}=0.001), \mathrm{HbA1c}(\mathrm{r}=-0.608, \mathrm{p}=0.001)$, total cholesterol $(\mathrm{r}=-0.872, \mathrm{P}=0.001)$, triglycerides $(\mathrm{r}=$ $0.862, \mathrm{p}=0.001)$, LDL $(\mathrm{r}=-0.777, \mathrm{p}=0.001)$, FBS $(\mathrm{r}=-0.907, \mathrm{p}=0.001)$ and $2 \mathrm{~h}$ PPS $(\mathrm{r}=-0.899$, $\mathrm{p}=0.001$ ), while there was a significant positive correlation between eGDR in T1DM patients and height SDS ( $r=0.610, p=0.001)$ and HDL ( $r=0.886, p=0.001)$ (Table 3). On the other hand, there was no correlation between eGDR and chronological age $(r=-0.248, p=0.101)$ or duration of disease $(r=-0.268, p=0.075)$ ( Table 3). Although mean $( \pm \mathrm{SD})$ SeP level, in this study, was higher in patients with T1DM than in controls $(59.78 \pm 60.05 \mathrm{ng} / \mathrm{ml}$ versus $55.57 \pm 7.6 \mathrm{ng} / \mathrm{ml})$, but with no significant $\mathrm{p}$ value $(\mathrm{P}=0.642)$ as shown in Table 2 .

In children with T1DM, there were significant positive correlations between $\mathrm{SeP}$ and duration of disease $(\mathrm{r}=0.413, \mathrm{P}=0.005), \mathrm{HDL}(\mathrm{r}=0.496, \mathrm{P}=0.001)$ and HbA1c $(\mathrm{r}=0.357, \mathrm{P}=0.016)$. Thus higher SeP was associated with poor controlled diabetes (Table 3). The Serum SeP levels of T1DM patients were negatively correlated significantly with their systolic and diastolic blood pressures with significant $\mathrm{p}$ value $(\mathrm{r}=-0.442, \mathrm{P}=0.002$ and $\mathrm{r}=-0.387, \mathrm{P}=0.009$ respectively) as shown in Table 3. On the other hand, the correlation between SeP levels of T1DM patients and their eGDR values was not significant $(r=0.149, \mathrm{P}=0.327)$, suggesting no association between SeP levels and IR as in table 3.

Table 3: Correlation between eGDR values and SeP level with demographic, clinical \& laboratory data of children and adolescents with T1DM patients.

\begin{tabular}{lcccc}
\hline & \multicolumn{2}{c}{ SeP } & \multicolumn{2}{c}{ eGDR } \\
\cline { 2 - 5 } & $\mathbf{r}$ & $\begin{array}{c}\text { P } \\
\text { value }\end{array}$ & $\mathbf{r}$ & P value \\
\hline Se p & & & 0.149 & 0.327 \\
\hline eGDR & 0.149 & 0.327 & & \\
\hline Age & 0.451 & 0.002 & -0.248 & 0.101 \\
\hline Duration of DM & 0.413 & 0.005 & -0.268 & 0.075 \\
\hline Weight SDS & -0.228 & 0.132 & -0.727 & 0.001 \\
\hline Height SDS & 0.248 & 0.100 & 0.610 & 0.001 \\
\hline BMI SDS & -0.319 & 0.051 & -0.883 & 0.001 \\
\hline Waist / Hip ratio & -0.255 & 0.091 & -0.746 & 0.001 \\
\hline Systolic percentile & -0.616 & 0.001 & -0.683 & 0.001 \\
\hline Diastolic percentile & -0.369 & 0.013 & -0.725 & 0.001 \\
\hline HbA1c & 0.357 & 0.016 & -0.608 & 0.001 \\
\hline Total cholesterol & -0.176 & 0.247 & -0.872 & 0.001 \\
\hline Triglycerides & -0.221 & 0.145 & -0.862 & 0.001 \\
\hline HDL & 0.346 & 0.020 & 0.886 & 0.001 \\
\hline LDL & -0.171 & 0.261 & -0.777 & 0.001 \\
\hline FBS & -0.039 & 0.800 & -0.907 & 0.001 \\
\hline 2h PPs & -0.080 & 0.601 & -0.899 & 0.001 \\
\hline
\end{tabular}

eGDR: estimated Glucose Disposal Rate; FBS: Fasting blood sugar; HDL: High density lipoprotein; LDL: Low density lipoprotein; $2 \mathrm{~h}$ PPs: $2 \mathrm{~h}$ post prandial sugar; P-value $<0.05$ : Statistically significant.

\section{Discussion}

Insulin resistance in T1DM patients is the result of various factors including genetic potential, exogenous insulin injection and life style (14). eGDR is a validated method for assessing insulin resistance in T1DM patients (13). eGDR of our studied cohort was reduced by $62 \%(7.78 \pm 3.08$ vs $12.53 \pm 0.91 \mathrm{mg} / \mathrm{kg} / \mathrm{min})$, compared to their age and gender matched controls. They had higher BMI and shorter stature. This noted overweight among TIDM children and adolescents seems to be universal, where reports of overweight estimates it to be $12.5 \%$ to $33.3 \%$ among children and adolescents with T1DM. This noted overweight was attributed to better glycemic control (15). Complications of this overweight among children with T1DM includes type 2 diabetes. Metabolic syndrome incidence complicates 3-29\% of children with T1DM who are overweight (16). Our study showed that T1DM patients with low eGDR, had higher systolic, diastolic blood pressure percentiles, HbA1c, total cholesterol, triglycerides, LDL, FBS, 2 h PPS, indicating insulin resistance in these patients and features of metabolic syndrome.

We have not assessed inulin receptor antibodies, but the noted decrement of eGDR suggests that our studied cohort suffered from insulin resistance. It is interesting however, that SeP did not correlate with eGDR. Hence, SeP cannot be used as a surrogate marker for insulin resistance. 
Patients with T1DM in this study were shorter than their age and gender matched controls. The shorter stature among our studied cohort might be related to the poor control of blood sugar, as their mean $\mathrm{HbA} 1 \mathrm{c}$ was very high (mean $\pm \mathrm{SD}=10.46 \pm 1.97 \%$ ). It is not clear if this was related to the hyperglycemia associated with the high HbA1c. We did not estimate their growth hormone levels, hence we are not aware if their short stature is related to growth hormone deficiency. Children with T1DM were reported to have deficiency in (GH) binding protein (17). GH receptors expression in the liver is regulated by insulin. Insulin therapy increases GH receptors but it does not normalize them (18).

In this study, patients who had low eGDR had higher weight SDS, BMI SDS and waist / hip ratio. This was similar to study conducted by Mihaela L. Bîcu et al who found that obese patients had lower eGDR in comparison with lean subjects (19). Higher insulin doses and an increase in the rate of hypoglycemia may be possible causes for development of obesity and subsequent insulin resistance in T1DM cases $(20)$.

Levels of selenoproteins are known to be affected by hepatic disease, dietary intake of selenium (21). In our study, SeP levels of T1DM patients did not differ significantly with that of the controls. This concurs with other studies. SeP levels did not correlate with insulin resistance in gestational diabetes (22). On the other hand, some studies reported progressive increase in serum SeP level in prediabetics and patients with T1DM (8). El-Kafrawy et al. showed in their study that obese and overweight had higher serum SeP concentrations than lean subjects (23). We have not estimated selenium intake in the diet of our studied cohort, so low selenium intake cannot be ruled out as a confounder of serum SeP.

$\mathrm{HbA1c}$ is a reliable marker for diabetic control that correlates well with both macro and micro vascular diabetic complications. In this study, serum SeP levels of the cases significantly correlated with duration of diabetes, HDL and HbA1c. Thus, it is probable that serum SeP level may be an indicator of diabetic control, but not of insulin resistance. The correlation between SeP and HbA1c has been reported by others as well (24), but is not unanimous, as lack of correlation was also reported. Others found either a negative correlation between SeP levels and HbA1c (25), or no significant correlation between the two variables (26). Hence, the serum SeP cannot be used as an indicator of insulin resistance.

Diabetic patients may have dyslipidemia; which increases their risk for development of early macrovascular complications. They may have high cholesterol, TG and low HDL. Low HDL may be a consequence for development of insulin resistance in these patients. Dyslipidemia should be screened in diabetic patients to prevent their development of early macrovascular complication $(27,28)$. Our study revealed that TIDM patients with high SeP, had high HDL. It seems that long-term selenium intake is associated with dyslipedemia irrespective of the glycemic control $(29,30)$.

In addition, we found that patients with low SeP had higher systolic and diastolic blood pressures percentiles. Similarly, Schomburg et al suggested in their study that low SeP levels may increase the risk for development of cardiovascular diseases. This can be explained by loss of the antioxidant properties of $\mathrm{SeP}(31)$.

Our study did not show significant relation between SeP level and eGDR value in T1DM patients. It rules out the sensitivity of $\mathrm{SeP}$ as a reliable indicator of insulin resistance. The small sample size does not allow studying the multifaceted effects of SeP, sensitivity and specificity of $\mathrm{SeP}$ as an indicator of adequate diabetic control. Larger numbers may shed more light on the role of SeP in diabetes mellitus. We did not assess selenium intake, or selenium nail content or measure the level of insulin antibodies in T1DM patients to be sure of reliability of the results. So we suggest further studies which should include measuring insulin antibodies level.

\section{Conclusion}

eGDR was a reliable measure for evaluation of IR in T1DM. T1DM and controls did not differ significantly in their serum SeP level. There was no significant relation between SeP and eGDR. Serum SeP level was not a reliable marker for insulin resistance in this study but may be indicator for diabetic control.

\section{Author Contributions:}

All authors shared in conceptualization, supervising, data curation, data analysis, writing original draft, data interpretation, writing original draft, supervising and revising. All authors reviewed the final manuscript. All authors have read and agreed to the published version of the manuscript. 


\section{FUNDING}

Authors declare there was no extramural funding provided for this study.

\section{CONFLICT OF INTEREST}

The authors declare no conflict of interest in connection with the study.

\section{References}

1. S. Tenny, M. Varacallo, Evidence Based Medicine. (StatPearls Publishing; Treasure Island (FL), 2020; https://www.ncbi.nlm.nih.gov/books/NBK470182/).

2. P. Bjornstad, Insulin sensitivity and complications in type 1 diabetes: New insights. World J. Diabetes. 6, 8 (2015).

3. C. S. Tam, W. Xie, W. D. Johnson, W. T. Cefalu, L. M. Redman, E. Ravussin, Defining Insulin Resistance From Hyperinsulinemic-Euglycemic Clamps. Diabetes Care. 35, 1605-1610 (2012).

4. E. J. Epstein, J. L. Osman, H. W. Cohen, S. N. Rajpathak, O. Lewis, J. P. Crandall, Use of the Estimated Glucose Disposal Rate as a Measure of Insulin Resistance in an Urban Multiethnic Population With Type 1 Diabetes. Diabetes Care. 36, 2280-2285 (2013).

5. H. Steinbrenner, B. Speckmann, A. Pinto, H. Sies, High selenium intake and increased diabetes risk: experimental evidence for interplay between selenium and carbohydrate metabolism. J. Clin. Biochem. Nutr. 48, 40-45 (2010).

6. E. Juszczuk-Kubiak, K. Bujko, M. Cymer, K. Wicińska, M. Gabryszuk, M. Pierzchała, Effect of Inorganic Dietary Selenium Supplementation on Selenoprotein and Lipid Metabolism Gene Expression Patterns in Liver and Loin Muscle of Growing Lambs. Biol. Trace Elem. Res. 172, 336-345 (2016).

7. V. M. Labunskyy, D. L. Hatfield, V. N. Gladyshev, Selenoproteins: Molecular Pathways and Physiological Roles. Physiol. Rev. 94, 739-777 (2014).

8. S. J. Yang, S. Y. Hwang, H. Y. Choi, H. J. Yoo, J. A. Seo, S. G. Kim, N. H. Kim, S. H. Baik, D. S. Choi, K. M. Choi, Serum Selenoprotein P Levels in Patients with Type 2 Diabetes and Prediabetes: Implications for Insulin Resistance, Inflammation, and Atherosclerosis. J. Clin. Endocrinol. Metab. 96, E1325-E1329 (2011).

9. Y. Saito, Selenoprotein $\mathrm{P}$ as a significant regulator of pancreatic $B$ cell function. J. Biochem. (Tokyo), mvz061 (2019).

10. World Medical Association, WMA Declaration of Helsinki- Ethical Principles for Medical Research Involving Human Subjects (2013), (available at https://www.wma.net/policiespost/wma-declaration-of-helsinki-ethical-principles-for-medical-research-involving-humansubjects/2013/).

11. D. M. Harrington, A. E. Staiano, S. T. Broyles, A. K. Gupta, P. T. Katzmarzyk, Waist circumference measurement site does not affect relationships with visceral adiposity and cardiometabolic risk factors in children: Waist circumference measurement site. Pediatr. Obes. 8, 199-206 (2013).

12. N. Ataei, M. Baikpour, M. Hosseini, M. Yousefifard, M. Fayaz, F. Ataei, A. Abbasi, Blood Pressure Nomograms for Children and Adolescents by Age and Body Mass Index in Tehran, Iran. Iran. J. Public Health. 46, 368-379 (2017).

13. A. Zabala, V. Darsalia, M. Lind, A.-M. Svensson, S. Franzén, B. Eliasson, C. Patrone, M. Jonsson, T. Nyström, Estimated glucose disposal rate and risk of stroke and mortality in type 2 diabetes: a nationwide cohort study. Cardiovasc. Diabetol. 20, 202 (2021).

14. G. Priya, S. Kalra, A Review of Insulin Resistance in Type 1 Diabetes: Is There a Place for Adjunctive Metformin? Diabetes Ther. 9, 349-361 (2018).

15. K. E. Minges, R. Whittemore, M. Grey, Overweight and Obesity in Youth With Type 1 Diabetes. Annu. Rev. Nurs. Res. 31, 47-69 (2013).

16. M. Grabia, R. Markiewicz-Żukowska, K. Socha, Prevalence of Metabolic Syndrome in Children and Adolescents with Type 1 Diabetes Mellitus and Possibilities of Prevention and Treatment: A Systematic Review. Nutrients. 13, 1782 (2021).

17. W. Bonfig, R. Holl, Mini Review/Commentary: Growth Hormone Treatment in Children with Type 1 Diabetes. Int. J. Mol. Sci. 20, 772 (2019).

18. A. Takano, T. Haruta, M. Iwata, I. Usui, T. Uno, J. Kawahara, E. Ueno, T. Sasaoka, M. Kobayashi, Growth Hormone Induces Cellular Insulin Resistance by Uncoupling 
Phosphatidylinositol 3-Kinase and Its Downstream Signals in 3T3-L1 Adipocytes. Diabetes. 50, 1891-1900 (2001).

19. M. L. Bîcu, D. Bîcu, S. Gârgavu, M. Sandu, M. I. Vladu, D. Clenciu, E. Moța, M. Moța, Estimated Glucose Disposal Rate (eGDR) - A Marker for the Assessment of Insulin Resistance in Type 1 Diabetes Mellitus. Romanian J. Diabetes Nutr. Metab. Dis. 23, 177182 (2016).

20. R. Nishtala, N. Kietsiriroje, M. Karam, R. A. Ajjan, S. Pearson, Estimated glucose disposal rate demographics and clinical characteristics of young adults with type 1 diabetes mellitus: A cross-sectional pilot study. Diab. Vasc. Dis. Res. 17, 147916412095232 (2020).

21. J. Avery, P. Hoffmann, Selenium, Selenoproteins, and Immunity. Nutrients. 10, 1203 (2018).

22. A. E. Altinova, O. T. Iyidir, C. Ozkan, D. Ors, M. Ozturk, O. Gulbahar, N. Bozkurt, F. B. Toruner, M. Akturk, N. Cakir, M. Arslan, Selenoprotein P is not elevated in gestational diabetes mellitus. Gynecol. Endocrinol. 31, 874-876 (2015).

23. N. A. F. El-Kafrawy, A. E.-B. M. Atta, S. Abdelsattar, S. K. E. D. Zewain, Serum selenoprotein $\mathrm{P}$ in lean and obese Egyptian individuals and its relation to insulin resistance. Alex. J. Med. 57, 61-69 (2021).

24. H. Misu, T. Takamura, H. Takayama, H. Hayashi, N. Matsuzawa-Nagata, S. Kurita, K. Ishikura, H. Ando, Y. Takeshita, T. Ota, M. Sakurai, T. Yamashita, E. Mizukoshi, T. Yamashita, M. Honda, K. Miyamoto, T. Kubota, N. Kubota, T. Kadowaki, H.-J. Kim, I. Lee, Y. Minokoshi, Y. Saito, K. Takahashi, Y. Yamada, N. Takakura, S. Kaneko, A Liver-Derived Secretory Protein, Selenoprotein P, Causes Insulin Resistance. Cell Metab. 12, 483-495 (2010).

25. M. Roman, A. Lapolla, P. Jitaru, A. Sechi, C. Cosma, G. Cozzi, P. Cescon, C. Barbante, Plasma selenoproteins concentrations in type 2 diabetes mellitus-a pilot study. Transl. Res. 156, 242-250 (2010).

26. B. Ilanbey, H. E. Yücel, C. Uçar, Ö. Kocamış, Selenoprotein P levels in patients with diabetes mellitus with complications. Int. J. Diabetes Dev. Ctries. (2021), doi:10.1007/s13410-02101029-0.

27. M. Femlak, A. Gluba-Brzózka, A. Ciałkowska-Rysz, J. Rysz, The role and function of HDL in patients with diabetes mellitus and the related cardiovascular risk. Lipids Health Dis. 16, 207 (2017).

28. B. Vergès, Pathophysiology of diabetic dyslipidaemia: where are we? Diabetologia. 58, 886899 (2015).

29. L. Su, S. Gao, F. W. Unverzagt, Y. Cheng, A. M. Hake, P. Xin, C. Chen, J. Liu, F. Ma, J. Bian, P. Li, Y. Jin, Selenium Level and Dyslipidemia in Rural Elderly Chinese. PLOS ONE. 10, e0136706 (2015).

30. W. Ju, M. Ji, X. Li, Z. Li, G. Wu, X. Fu, X. Yang, X. Gao, Relationship between higher serum selenium level and adverse blood lipid profile. Clin. Nutr. 37, 1512-1517 (2018).

31. L. Schomburg, M. Orho-Melander, J. Struck, A. Bergmann, O. Melander, Selenoprotein-P Deficiency Predicts Cardiovascular Disease and Death. Nutrients. 11, 1852 (2019).

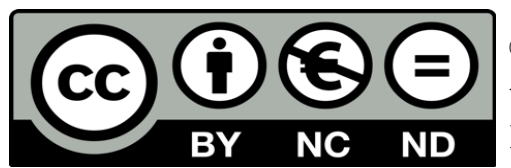

(c) 2021 submitted by the authors. Open access publication under the terms and conditions of the Creative Commons Attribution (CC- BYNC- ND) license. (https://creativecommons.org/licenses/by-nc-nd/2.0/). 